\title{
Effective Modified Carbon Nanofibers as Electrodes for Capacitive Deionization Process
}

\author{
Nasser A. M. Barakat ${ }^{1,2 *}$, Ahmed G. El-Deen ${ }^{3}$, Khalil Abdelrazek Khalil ${ }^{4}$ \\ ${ }^{1}$ Organic Materials and Fiber Engineering Department, Chonbuk National University, Jeonju, South Korea \\ ${ }^{2}$ Chemical Engineering Department, Faculty of Engineering, Minia University, El-Minia, Egypt \\ ${ }^{3}$ BioNanosystem Department, Chonbuk National University, Jeonju, South Korea \\ ${ }^{4}$ Mechanical Engineering Department, (NPST), King Saud University, Riyadh, Saudi Arabia \\ Email: "nasser@jbnu.ac.kr
}

Received November 2013

\begin{abstract}
Carbon materials have the advantages of good electrical conductivity and excellent chemical stability, so many carbon materials have been introduced as electrodes for the capacitive deionization (CDI) process. Due to the low surface area compared to the other nanocarbonaceous materials, CNFs performance as electrode in the CDI units is comparatively low. This problem has been overcome by preparing high surface area carbon nanofibers and by creating numerous long pores on the nanofibers surface. The modified CNFs have been synthesized using low cost, high yield and facile method; electrospinning technique. Stabilization and graphitization of electrospun nanofiber mats composed of polyacrylonitrile (PAN) and poly (methyl methacrylate) (PMMA) leads form longitudinal pores CNFs. The utilized characterizations indicated that the CNFs obtained from electrospun solution having 50\% PMMA have surface area of $181 \mathrm{~m}^{2} / \mathrm{g}$ which are more than the conventional CNFs. Accordingly, these nanofibers revealed salt removal efficiency of $\sim 90 \%$ and specific capacitance of $237 \mathrm{~F} / \mathrm{g}$.
\end{abstract}

\section{KEYWORDS}

Capacitive Deionization; Carbon Nanofibers; Electrospinning; Multi-Channels CNFs; Desalination

\section{Introduction}

In the last four decades, the number and capacities of desalination units have increased dramatically; 45\% Multi-Stage Flash (MSF) and 42\% Reverse Osmosis (RO) of world capacity [1]. Capacitive deionization (CDI) is a promised desalination technology due to power saving, low operation and maintenance costs [2], moreover no chemicals are used in pre- or post-treatment processes; so CDI is considered green and economic desalination technology [3]. Many carbon materials have been introduced as electrodes for the CDI process such as carbon aerogels [4,5], activated carbon (AC) [6,7], activated carbon fiber (ACF) $[8,9]$, carbon nanotubes (CNTs) [10], and Graphene [11-13]. Carbon nanfibres (CNFs) prepared by the electrospinning process gradually attracted the attention of most researchers because of the associated advantages of the synthesizing technique and the obtained product. The long axial ratio of the CNFs leads to decrease the total surface area compared to the other nanostructural carbonaceous materials, this might have negative influ-

"Corresponding author. ence upon utilizing CNFs as electrodes in the CDI process. However, recently it was reported that the long axial ratio provides advantage for the nanofibers over the nanoparticles in the electrons transfer-based processes as the nanofibers reveal better performances [14]. In this study, the surface area of the CNFs has been modified by producing multi channeled CNFs. Typically, electrospinning of binary polymer solution composed of PAN and poly (methyl methacrylate) (PMMA) was achieved. Due to the big difference in the thermal properties between the two polymers as PMMA is completely eliminated during the carbonization step [15], the physiochemical characterization indicated that the surface area strongly increases with increasing the PMMA content which positively affects the performance as the nanofibers prepared from PAN/PMMA solution having 50 wt\% PMMA reveal higher ions electrosportion compared to the other formulations.

\section{Experimental}

\subsection{Materials}

Polyacrylonitrile $\left(\mathrm{PAN}\right.$, average $\mathrm{Mw}=150000 \mathrm{~g} \cdot \mathrm{mol}^{-1}$ ) 
and poly(methyl methacrylate) (PMMA, average $\mathrm{Mw}=$ $120000 \mathrm{~g} \cdot \mathrm{mol}^{-1}$ ) are of analytical grade and were purchased from Sigma-Aldrich. N,N-dimethylformamide (DMF 99.5\% assay; SAMCHUN Pure Chemical Co., South Korea) without any further modifications was used as a solvent..

\subsection{Preparation of Multi-Channels Carbon Nanofibers}

First, PAN and PMMA solutions (10 wt $\%$ in DMF) were prepared individually in two glass bottles using ultrasonication for $2 \mathrm{~h}$ then heating with stirring in water bath at $60^{\circ} \mathrm{C}$ for $4 \mathrm{~h}$. Electrospun solutions having different PMMA:PAN ratios were prepared. Typically, PMMA mass percentages with respect to PAN were 0,25 and $50 \%$. The bipolymer solutions were further ultrasconicated for $0.5 \mathrm{~h}$. Electrospinning of these solutions was carried out at $20 \mathrm{kV}$ and $18 \mathrm{~cm}$ distance between the collector and the tip of the syringe. The oxidative stabilization was carried out at $280^{\circ} \mathrm{C}$ for $1 \mathrm{~h}$ with heating rate 5 $\mathrm{deg} / \mathrm{min}$ and the carbonization was done at $1000^{\circ} \mathrm{C}$ under argon atmosphere for $5 \mathrm{~h}$ with a rate $5 \mathrm{deg} / \mathrm{min}$.

\subsection{Characterizations}

The surface morphology was studied by a JEOL JSM5900 scanning electron microscope (JEOLLtd., Japan) and field-emission scanning electron microscope (FESEM Hitachi S-7400, Japan). High resolution image and selected area electron diffraction patterns were obtained with transmission electron microscope (TEM, JEOL JEM-2010, Japan) operated at $200 \mathrm{kV}$. Cyclic voltammetry measurement was carried out using different concentrations (0.1, 0.5 and $1 \mathrm{M}) \mathrm{NaCl}$ solutions and the sweep potential rang was adjusted from -0.4 to $0.6 \mathrm{~V}$ in electrochemical cell with three-electrode system: platinum wire as counter electrode, $\mathrm{Ag} / \mathrm{AgCl}$ as reference electrode and the prepared materials as working electrode. This system was controlled using VersaStat4 potentiostat device with through in VersaStudio software program.

\section{Results and Discussion}

Simplicity of the electrospinning process, the diversity of the electrospinnable materials, and the unique features of the obtained electrospun nanofibers provide especial interest for both of the technique and the resultant products. The past decades have witnessed tremendous progress in the development of the electrospinning process to widen the applications of the obtained products. Most of the reported functional inorganic nanofibers have been prepared using the electrospinning techniques. Good morphology nanofibers were obtained from electrospinning of PMMA/PAN polymer blends. Figure 1 displays the

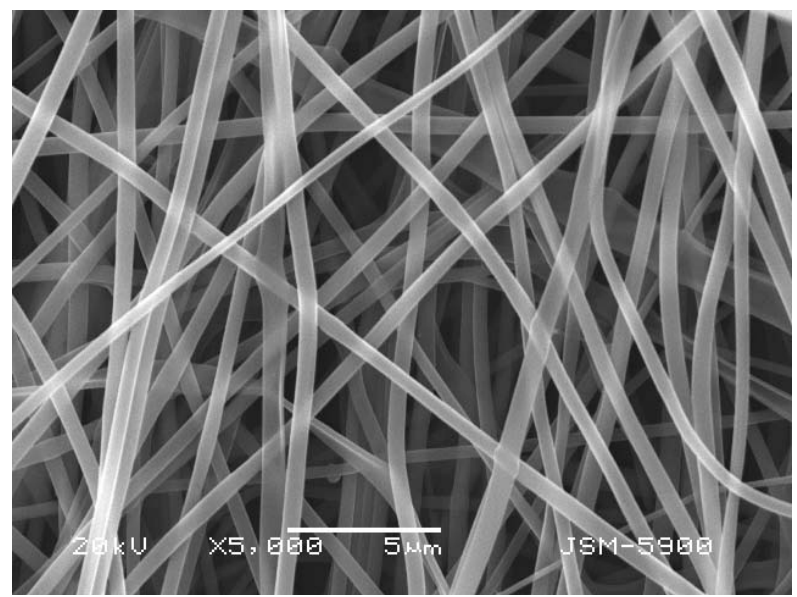

Figure 1. SEM image for the PAN/PMMA electrospun nanofibers.

electrospun nanofibers obtained from the prepared PMMA/PAN solutions, as shown in the figure good morphology nanofibers have been obtained; continuous and beads-free nanofibers are observed. It is noteworthy mentioning that the diameters frequency chart has been estimated. From the obtained data, the average diameters have been estimated to be 519, 335 and $226 \mathrm{~nm}$ for the electrospun nanofiber mats prepared from the solutions containing 0, 25 and $50 \mathrm{wt} \%$ PMMA with respect to PAN, respectively.

Figure 2 shows the FE SEM images after stabilization and graphitization processes, the corresponding average diameters have been estimated by the same methodology; the results indicated that the average diameters are 382, 220 and $160 \mathrm{~nm}$ for the sintered nanofibers prepared from the solutions containing 0, 25 and $50 \mathrm{wt} \%$ PMMA with respect to PAN, respectively. Interestingly, the nanofibers synthesized from the last formulation have many channels on the surface. This finding clearly appears in the FE SEM and TEM images. Formation of the observed channels can be explained from the nature of the PMMA/PAN solution. This polymer-blend solution has phase separation which results in sea-islands feature occurs due to the intrinsic properties (e.g., interfacial tension, viscosity, elasticity) of the two polymers [16]. Therefore, the prepared solutions can be considered as stable emulsion-like polymer-blend solutions. The continuous phase consisted of PAN solution and the homogeneously dispersed phase consisted of PMMA solution. It is noteworthy mentioning that the viscosity and surface tension of the PMMA/PAN solutions were intensively studied by other authors [16]. The obtained results indicated that the viscosity increased when the volume fraction of PAN in the blend solution increased. The surface tension of the PAN solution $\left(5.195 \mathrm{mNm}^{-1}\right)$ was found to be higher than that of the PMMA solution $\left(8.843 \mathrm{mNm}^{-1}\right)$. Thus, the surface tension of the constituent polymers in 

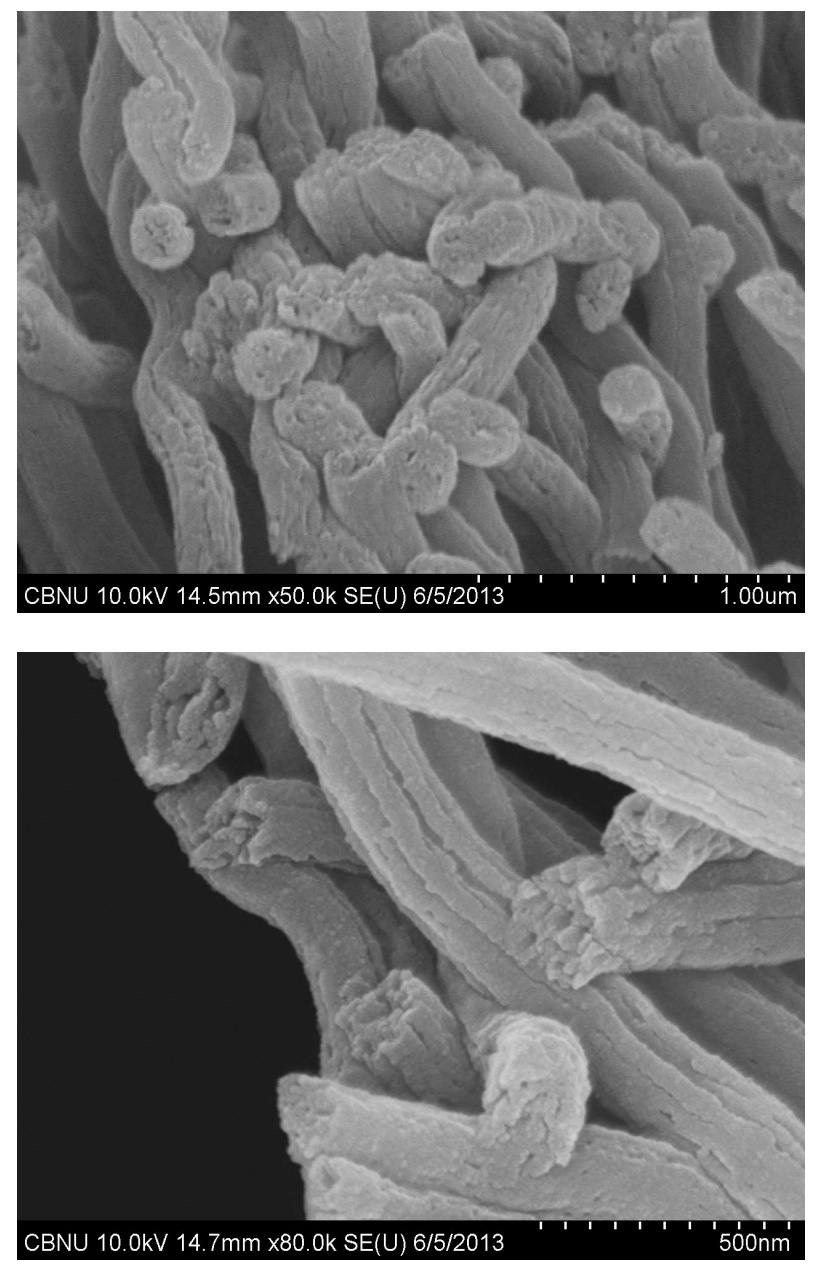

Figyre 2. FE SEM images for the graphitized PAN/PMMA electrospun nanofibers; PMAA $=\mathbf{5 0} \%$.

the blend solution is the most important factor in determining the surface morphology of the electrospun organic nanofibers. As a result, the low-surface-tension polymer (PAN) occupies the continuous phase of the solution while the high-surface-tension polymer (PMMA) forms the discontinuous phase.

Cyclic voltammetry (CV) measurements are often applied as effective tools to characterize the CDI performance of electrodes. Figure 3 depicts CV curves of the CNFs, reduced graphene oxide (rGO) and multi-channels carbon nanofibers MC-CNFs $25 \%$, and MC-CNFs 50\% electrodes at sweep rates 10,50 and $100 \mathrm{mV} \cdot \mathrm{s}^{-1}$ in 0.1 , 0.5 , and $1 \mathrm{M} \mathrm{NaCl}$ solution. As shown in the figure, the MC-CNFs $50 \%$ clearly retain a rectangular shape over a wide range of applied voltage. Obviously, typical rectangular profile implies excellent electrochemical doublelayer capacitance and also indicates highly reversible and reliable electrosorption charge-discharge process [17]. Furthermore, MC-CNFs 50\% nanofibers showed precedence at high concentration $\mathrm{NaCl}$ solutions with high electrosorption capacity which indicates hard saturation
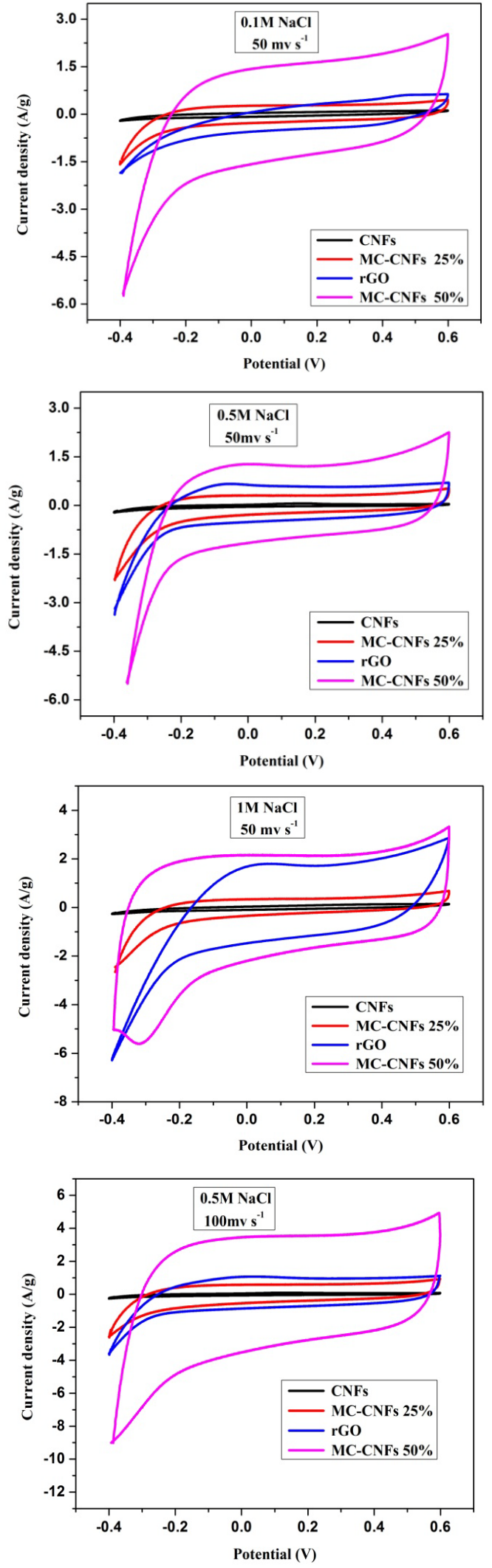

Figure 3. Cyclic voltammetry results for the prepared materials at different $\mathrm{NaCl}$ concentration and various sweep rates. 
of the internal mesoporous and systematic channel structure. Another important finding can be observed from Figure 3 is that the performances of the investigated materials are linearly related in the estimated surface areas which support our original target about improving the surface area of the CNFs. It is noteworthy mentioning that the MC-CNFs $50 \%$ nanofibers reveal the best performance at all the scan rates and for all the investigated $\mathrm{NaCl}$ solution concentrations. As aforementioned in the experimental section, the specific capacitances have been estimated from I-V cycles. Figures 3(c) and (d) display the cyclic voltammetery measurements for the prepared materials at various scan rates and $0.5 \mathrm{M} \mathrm{NaCl}$ solution.

Graphene has been introduced as extremely promising electrode for capacitive deionization [11-13]. Graphene is an intriguing 2D monolayer carbon sheet whose distinct properties make it very promising in desalination applications. Since the first report of graphene production by mechanical exfoliation method, many techniques broadly classified as top down and bottom up syntheses have been developed. Among the various reported methods for producing graphene, chemical reduction of graphite oxide (GO), or chemically converted graphene, provides not only an established, low-cost and scalable approach, but also a highly flexible method for the chemical fictionalization of graphene materials $[18,19]$. Therefore, in this study, this chemical rout was invoked. An important property of GO, brought about by the hydrophilic nature of the oxygenated graphene layers, is its easy exfoliation in aqueous media. As a result, GO readily forms stable colloidal suspensions of thin sheets in water [20]. After a suitable ultrasonic treatment, such exfoliation can produce stable dispersions of very thin graphene oxide sheets in water [18]. Therefore, colloidal suspension of the prepared GO prepared with the aid of ultrasound was clear, homogenous, and stable. It is known that with increasing the scan rate the specific capacitance dramatically decreases. As shown in Figure 4, the corresponding specific capacitance of the introduced MC-CNFs $50 \%$ is higher than the other carbonaceous materials. Typically, at $10 \mathrm{mV} / \mathrm{s}$ scan rate, the specific capacitances are 235, 188, 162 and $63 \mathrm{~F} / \mathrm{g}$ for the MCCNFs $50 \%$, rGO, MC-CNFs $25 \%$ and CNFs, respectively. Another interesting finding is that the introduced materials display good specific capacitance stability at the high scan rates which is a good feature for the carbonaceous materials making them adequate to be utilized as electrodes in the CDI units. It is noteworthy mentioning that the electrosorption capacitance is generally high at lower scan rates because that the diffusion of ions from the solution could gain more available access to the electrode surface which leads to more surface adsorption/desorption of ions [21]. However, at high scan rates, the effective inner surface adsorption of ions would be

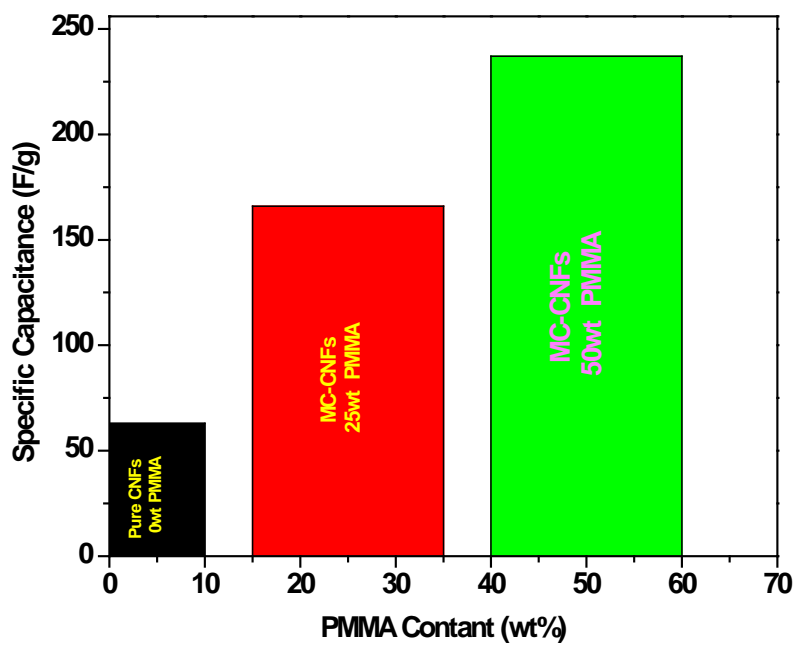

Figure 4. Effect of PMMA content on the specific capacitance of the introduced CNFs.

reduced accordingly. The desalination performance behavior of the synthesized materials was determined by using the CDI cell in a $\mathrm{NaCl}$ aqueous solution that has an initial conductivity of $\sim 73 \mu \mathrm{S} / \mathrm{cm}$. Obviously, rapid decrease in the conductivity in early stage reflects distinct change in the $\mathrm{NaCl}$ concentration in CDI cell which indicates the capability of the investigated material towards ionic adsorption/desorption process. The desalination performance curve illustrates the salt removal efficiency ( $\eta$ ) of the MC-CNFs $50 \%$ has a great proportion reach to 89.04\% compared to CNFs, MC-CNFs $25 \%$ and rGO which have desalination efficiencies of $56.57 \%$, $73.68 \%$, $82.89 \%$, respectively. This improvement in salt removal can be attributed to the propitious internal pore structure which facilitates the ions adsorption from the solution to channel punctures on electrode. Furthermore, the MCCNFs $50 \%$ have the highest electrosorptive capacity (2.21 mg/g) compared to the other formulations because of created channels on the surface of introduced modified carbon nanofibers.

\section{Conclusion}

Polyacrylonitrile (PAN) and poly (methyl methacrylate) (PMMA) in DMF form stable polymer blend solution. Electrospinning of the PAN/PMMA biopolymer solution leads to produce smooth and beads-free nanofibers. Because of the complete thermal decomposition of PMMA within the temperature range of the graphitization process, the electrospun mats containing $50 \mathrm{wt} \%$ PMMA with respect to PAN reveals carbon nanofibers having many channels on the surface. The proposed modified CNFs have adsorption and desorption ability of ions salt more than reduced graphene oxide and normal CNFs. In addition, the introduced CNFs exhibit superiority in specific capacitance value. Therefore, the modified carbon 
nanofibers can be successfully utilized as electrodes in capacitive deionization units.

\section{Acknowledgements}

This work was financially supported by the National Plan for Science \& Technology (NPST), King Saud University Project No. 11-NAN1460-02.

\section{REFERENCES}

[1] A. D. Khawaji, I. K. Kutubkhanah and J. M. Wie, "Advances in Seawater Desalination Technologies," Desalination, Vol. 221, 2008, pp. 47-69. http://dx.doi.org/10.1016/j.desal.2007.01.067

[2] M. A. Anderson, A. L. Cudero and J. Palma, "Capacitive Deionization as an Electrochemical Means of Saving Energy and Delivering Clean Water. Comparison to Present Desalination Practices: Will It Compete?” Electrochim Acta, Vol. 55, 2010, pp. 3845-3856. http://dx.doi.org/10.1016/j.electacta.2010.02.012

[3] T. Welgemoed and C. Schutte, "Capacitive Deionization Technology ${ }^{\mathrm{TM}}$ : An Alternative Desalination Solution,” Desalination, Vol. 183, 2005, pp. 327-340. http://dx.doi.org/10.1016/j.desal.2005.02.054

[4] J. C. Farmer, D. V. Fix, G. V. Mack, R. W. Pekala and J. F. Poco, "Capacitive Deionization of $\mathrm{NaCl}$ and $\mathrm{NaNO}_{3}$ Solutions with Carbon Aerogel Electrodes," Journal of The Electrochemical Society, Vol. 143, 1996, pp. 159-169. http://dx.doi.org/10.1149/1.1836402

[5] C. M. Yang, W. H. Choi, B. K. Na, B. W. Cho and W. I. Cho, "Capacitive Deionization of $\mathrm{NaCl}$ Solution with Carbon Aerogel-Silica Gel Composite Electrodes,” Desalination, Vol. 174, 2005, pp. 125-133. http://dx.doi.org/10.1016/j.desal.2004.09.006

[6] L. Zou, G. Morris and D. Qi, "Using Activated Carbon Electrode in Electrosorptive Deionisation of Brackish Water," Desalination, Vol. 225, 2008, pp. 329-340. http://dx.doi.org/10.1016/j.desal.2007.07.014

[7] L. Pan, X. Wang, Y. Gao, Y. Zhang, Y. Chen and Z. Sun, "Electrosorption of Anions with Carbon Nanotube and Nanofibre Composite Film Electrodes,” Desalination, Vol. 244, 2009, pp. 139-143.

http://dx.doi.org/10.1016/j.desal.2008.05.019

[8] G. Wang, Q. Dong, Z. Ling, C. Pan, C. Yu and J. S. Qiu, "Hierarchical Activated Carbon Nanofiber Webs with Tuned Structure Fabricated by Electrospinning for Capacitive Deionization,” Journal of Materials Chemistry, Vol. 22, 2012, pp. 21819-21823. http://dx.doi.org/10.1039/c2jm34890j

[9] G. Wang, C. Pan, L. P. Wang, Q. Dong, C. Yu, Z. B. Zhao and J. S. Qiu, “Activated Carbon Nanofiber Webs Made by Electrospinning for Capacitive Deionization,” Electrochim Acta, Vol. 69, 2012, pp. 65-70. http://dx.doi.org/10.1016/j.electacta.2012.02.066

[10] L. Wang, M. Wang, Z. H. Huang, T. X. Cui, X. C. Gui, F. Y. Kang, K. L. Wang and D. H. Wu, "Capacitive Deionization of $\mathrm{NaCl}$ Solutions Using Carbon Nanotube Sponge Electrodes,” Journal of Materials Chemistry, Vol. 21,
2011, pp. 18295-18299.

http://dx.doi.org/10.1039/c1jm13105b

[11] D. S. Zhang, T. T. Yan, L. Y. Shi, Z. Peng, X. R. Wen and J. P. Zhang, "Enhanced Capacitive Deionization Performance of Graphene/Carbon Nanotube Composites,” Journal of Materials Chemistry, Vol. 22, 2012, pp. 14696-14704. http://dx.doi.org/10.1039/c2jm31393f

[12] H. B. Li, L. K. Pan, C. Y. Nie, Y. Liu and Z. Sun, "Reduced Graphene Oxide and Activated Carbon Composites for Capacitive Deionization,” Journal of Materials Chemistry, Vol. 22, 2012, pp. 15556-15561. http://dx.doi.org/10.1039/c2jm32207b

[13] H. B. Li, L. D. Zou, L. K. Pan and Z. Sun, "Novel Graphene-Like Electrodes for Capacitive Deionization,” Environmental Science \& Technology, Vol. 44, 2010, pp. 8692-8697. http://dx.doi.org/10.1021/es101888j

[14] N. A. Barakat, M. A. Kanjawal, I. S. Chronakis and H. Y. Kim, "Influence of Temperature on the Photodegrdation Process Using Ag-Doped $\mathrm{TiO}_{2}$ Nanostructures: Negative Impact with the Nanofibers," Journal of Molecular Catalysis A: Chemical, Vol. 336, 2012, pp. 333-340.

[15] C. Kim, Y. I. Jeong, B. T. N. Ngoc, K. S. Yang, M. Kojima, Y. A. Kim, M. Endo and J. W. Lee, "Synthesis and Characterization of Porous Carbon Nanofibers with Hollow Cores through the Thermal Treatment of Electrospun copolymeric Nanofiber Webs,” Small, Vol. 3, 2006, pp. 91-95. http://dx.doi.org/10.1002/smll.200600243

[16] C. Kim, Y. I. Jeong, B. T. N. Ngoc, K. S. Yang, M. Kojima, Y. A. Kim, M. Endo and J. W. Lee, "Synthesis and Characterization of Porous Carbon Nanofibers with Hollow Cores through the Thermal Treatment of Electrospun Copolymeric Nanofiber Webs,” Small, Vol. 3, 2007, pp. 91-95. http://dx.doi.org/10.1002/smll.200600243

[17] C.-H. Hou and C.-Y. Huang, "A Comparative Study of Electrosorption Selectivity of Ions by Activated Carbon Electrodes in Capacitive Deionization,” Desalination, Vol. 314, 2013, pp. 124-129. http://dx.doi.org/10.1016/j.desal.2012.12.029

[18] S. Stankovich, D. A. Dikin, R. D. Piner, K. A. Kohlhaas, A. Kleinhammes, Y. Jia, Y. Wu, S. B. T. Nguyen and R. S. Ruoff, "Synthesis of Graphene-Based Nanosheets via Chemical Reduction of Exfoliated Graphite Oxide," Carbon, Vol. 45, 2007, pp. 1558-1565. http://dx.doi.org/10.1016/j.carbon.2007.02.034

[19] Y. Zhu, S. Murali, W. Cai, X. Li, J. W. Suk, J. R. Potts and R. S. Ruoff, "Graphene and Graphene Oxide: Synthesis, Properties, and Applications,” Advanced Materials, Vol. 22, 2010, pp. 3906-3924. http://dx.doi.org/10.1002/adma.201001068

[20] S. Stankovich, R. D. Piner, X. Chen, N. Wu, S. T. Nguyen and R. S. Ruoff, "Stable Aqueous Dispersions of Graphitic Nanoplatelets via the Reduction of Exfoliated Graphite Oxide in the pResence of Poly(sodium 4-styrenesulfonate)," Journal of Materials Chemistry, Vol. 16, 2006, pp. 155-158. http://dx.doi.org/10.1039/b512799h

[21] L. Zou, L. Li, H. Song and G. Morris, "Using Mesoporous Carbon Electrodes for Brackish Water Desalination,” Water Research, Vol. 42, 2008, pp. 2340-2348. http://dx.doi.org/10.1016/j.watres.2007.12.022 